\title{
Performance of Voice Calls with Rate Matching Optimization in UMTS Networks
}

\author{
Eduardo R. Vale, João Célio B. Brandão, Marco Grivet
}

\begin{abstract}
Two transmission modes were standardized in UMTS: a Circuit Switched (CS) and a Packet Switched (PS) mode. In both cases, an efficient transmission framework was defined to allow the transmission of multimedia information with the required reliability, in spite of the medium characteristics. Multimedia calls are delivered in several transport channels - one for each media related to the call - properly multiplexed in one or more physical channels. These transport channels are designed to attain the required levels of QoS using two main techniques: Channel Coding and Rate Matching. In this paper we discuss the creation of transport channels for voice calls in both CS and PS modes and present a new methodology to optimize the Rate Matching parameters for a given channel coder and a set of QoS objectives. With the proposed methodology the maximum $E_{b} / N_{0}$ assigned to the transport channels is minimized, thus reducing the transmitted power.
\end{abstract}

Index Terms-UMTS, Rate Matching, Power control, Optimization

\section{INTRODUCTION}

In recent years, Internet and cellular applications have emerged as the major driving forces behind new developments in the area of telecommunications networks. Furthermore, there is a great interest in using IP technology to provide multimedia services in general.

Cellular systems are evolving from a purely telephony system to a multimedia service based on the IP technology which has been incorporated in $3 \mathrm{G}$ and particularly in the UMTS specifications [1],[2]. In fact, the UMTS system is in continuing evolution. There have been several releases, each of them providing a new version of specifications for vendors and operators to be used in system implementation. Presently, a reference architecture specifies the main network components of UMTS including interfaces between access network, core network and PSTN and also signaling network.

Two domains were delimited in the UMTS architecture: a Circuit Switched (CS) and a Packet Switched (PS) domains. 3GPP Release 5 introduced the IMS-(IP Multimedia Subsystem), intended to support multimedia traffic in general.

The main difference between UMTS and the second generation mobile CDMA system is the ability to offer simultaneous flexible services at various rates as high as $2 \mathrm{Mbit} / \mathrm{s}$ (in dedicated channels), that were not possible in the conventional cellular system. These services consist of voice, video, circuit switched data and packet switched data services. Different

Eduardo R. Vale is with Telecommunication Department of Universidade Federal Fluminense. E-mail: eduardo.vale@globo.com

João Célio B. Brandão and Marco Grivet are with Center of Telecommunication Studies of Pontifical Catholic University of Rio de Janeiro. E-mails: jcelio@cetuc.puc-rio.br,mgrivet@cetuc.puc-rio.br combinations of multiple services are offered by using multiple transport channels, each having their own characteristics such as CRC code length, channel coding schemes and coding rate. One very important part in UMTS that is responsible to provide flexible services is the Rate Matching process that is part of the transport channel coding and multiplexing mechanism. When combining two or more different services, it is necessary to adjust the channel transmission parameters in order to fulfill different Quality of Service (QoS) requirements. To sort this matter out, the Rate Matching unit is used to repeat or puncture coded bits, in order to achieve the necessary balancing effect.

Several papers have been published seeking to clarify, detail or discuss the UMTS next-generation architecture, in particular, the all IP scenario [3],[4]. A general description of the present UMTS architecture may be found in [5]. Concerning Rate Matching, there are several papers in the literature [6],[7],[11] considering the subject, but none of them gives a reasonable treatment to the aspect of optimization process. Furthermore, 3GPP does not consider any optimization methodology in the standardization documents related to the Rate Matching unit.

Here, we intend to address some important aspects related to the performance of voice calls in UMTS. We introduce a discussion on the relative performance of the end-to-end voice calls developed in CS and PS modes considering the air interface effect and the heavy signaling burden in the PS mode. In this context we focus on the rate matching process for which we propose an optimization procedure. This is capable to give the exact number of bits to repeat or puncture, in order to achieve the best commitment between the maximum required power for transmission and the required levels of QoS for each service. To the best of the author's knowledge, the approach here investigated to optimally allocate repetition/puncturing bits has not yet been addressed in the technical literature.

This paper is organized as follows: Section 2 discusses the creation and multiplexing of transport channels. Section 3 presents the voice transmission process in CS and PS modes. Section 4 briefly describes the Rate Matching process while Section 5 formally introduces the related mathematical formulation for the optimization problem which leads to a set of features for the optimal solution. Section 6 shows two application examples based on practical cases related to UMTS specifications and finally section 7 presents the conclusions.

\section{CReation And Multiplexing of Transport Channels}

The UMTS system is capable to simultaneously work with several media at varying rates and different reliability levels. 
With the concept of "transport channels", the information to be transmitted is conveniently allocated to one or more transport channels, each with its proper QoS level. Both the Circuit Switched (CS) and the Packet Switched (PS) modes use this concept.

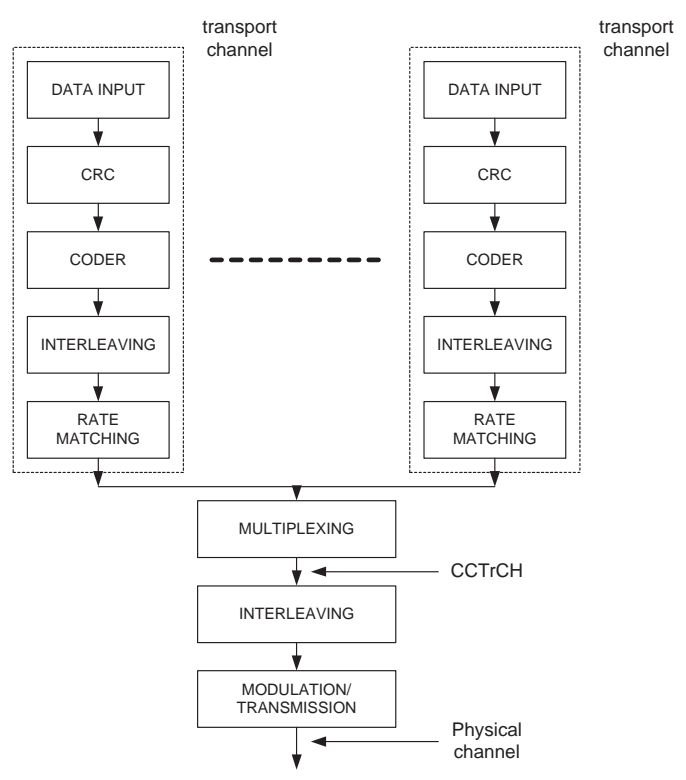

Fig. 1. UMTS Communication System

Figure 1 shows the process of creation and multiplexing of transport channels in the case of uplink transmission [8]. For each channel, after the CRC attachment, the data blocks are either concatenated or segmented, in order to fit the allowable limits specified for the block sizes. Next, the channel encoding is performed, with four different coding possibilities: $1 / 2$ convolutional coding (CC2), 1/3 convolutional coding (CC3), $1 / 3$ turbo coding (TC) or no coding. The RF equalization and segmentation have the purpose of adapting the data to the radio frames, when more than one radio frame is transmitted. Interframe interleaving is then performed on a time window that spans one, two, four or eight radio frames of $10 \mathrm{~ms}$ duration. Next, the Rate Matching block performs two processes:

- First, the sum of the instantaneous bit rates of all transport channels is adapted to the physical channel capacity using bits repetition or puncturing;

- Second, the mechanism of repetition and puncturing is used to differentiate the channels in respect to individual QoS needs. For example, in one situation, the channels with more stringent requirements will have assigned more repetition bits;

Every $10 \mathrm{~ms}$, the rate matched outputs of all the transport channels are multiplexed, forming the Coded Composite Transport Channel $(\mathrm{CCTrCH})$. After intra-frame interleaving and the modulation phase, the $\mathrm{CCTrCH}$ is allocated for transmission in one or more physical channels [8]. Note that the physical channel bit rate is determined by CDMA spread factor which may assume values from 4 to 512 .

\section{VoICE TRANSMission In CS AND PS MOdES}

In this section the process previously described will be detailed for the case of voice transmission in PS and CS modes of a FDD UMTS system using the AMR codec at $12.2 \mathrm{kbit} / \mathrm{s}$.

In CS mode we have a total of four channels, as defined in [9]:

- Channel A: Carrying the most sensitive information bits (81 bits) of the AMR codec, with Error Probability (EP) of $5.10^{-4}$. The $1 / 3$ Convolutional Code (CC3) is used;

- Channel B: Carrying the medium sensitive information bits (103 bits), with Error Probability (EP) of $10^{-3}$. The 1/3 Convolutional Code (CC3) is used;

- Channel $C$ : Carrying the least sensitive information bits (60 bits), with Error Probability (EP) of $5.10^{-3}$. The $1 / 2$ Convolutional Code (CC2) is used;

- Channel D: For signalization purposes (148 bits), with Error Probability (EP) of $10^{-4}$. The $1 / 3$ Convolutional Code (CC3) is used;

Figure 2 illustrates the frame structure for these transport channels (a spread factor of 64 is assumed). The tail bits are used for the initialization of the respective coders. Note that the final number of bits depends of the Rate Matching unit.

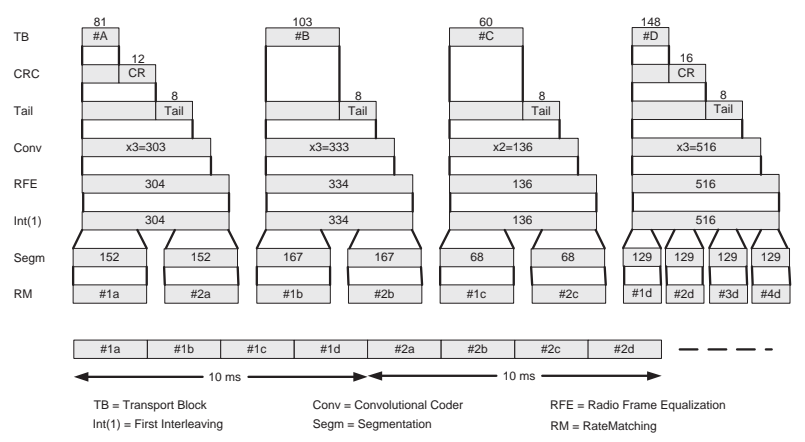

Fig. 2. Transmission of voice signal (CS mode)

In PS mode it is necessary to transmit packets with varying sizes and other different characteristics. 3GPP is currently developing an adequate support for the transmission of the following different categories of packets:

- RTP (Real Time Transport Protocol) voice packets with no header compression (99 bytes): these occur at the beginning of the call and whenever it is necessary to adjust the mechanism of header compression (ROHC Robust Header Compression);

- RTP voice packets with header compression (40 bytes): they constitute the major part of the voice packet transmission;

- RTCP (Real Time Transport Control Protocol) packets with no header compression (140 bytes): they occur not very frequently, during the realization of the call;

- SIP packets, possibly with header compression (until 7500 bytes: they occur mostly at the beginning and the end of the call; 
- Voice call signaling packets: for telephony signaling purpose; they carry the same information of the channel D specified before;

For the sake of simplicity, let us consider a VoIP transmission with the most favorable condition, i.e. voice packets with header compression, simultaneously with voice call signaling packets. Figure 3 describes this situation.

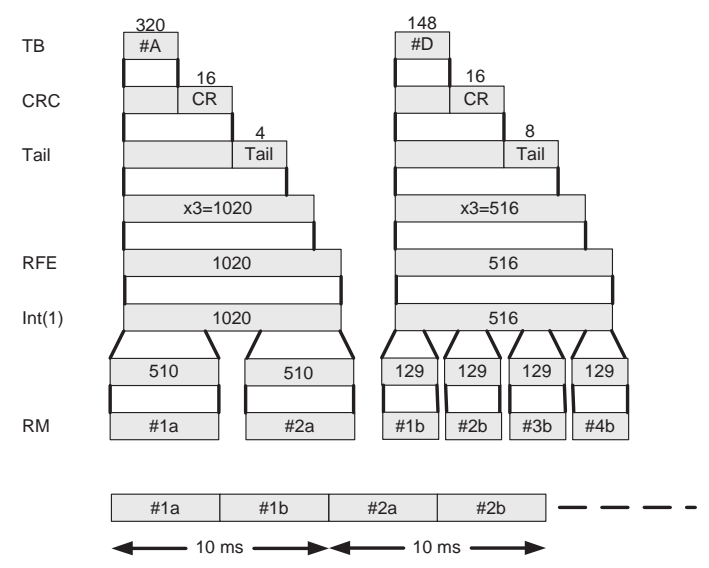

Fig. 3. Transmission of voice signal (PS mode)

Here, we have channel A carrying voice multiplexed with channel B (like channel D of CS mode). Before the rate matching unit we have 639 bits $(510+129)$ to be accommodated in the physical channel. There are two options:

- The first one is to use a spread factor of 32, corresponding to the rate of $120 \mathrm{kbit} / \mathrm{s}$. Then, to fit $639 \mathrm{bits}$ in a radio frame of $10 \mathrm{~ms}$ (1200 bits) we will have 561 bits (1200639) to use as repetition bits, distributed over the two channels;

- The second one is to use a spread factor of 64 (like the CS case), corresponding to the rate of $60 \mathrm{kbit} / \mathrm{s}$. Now we will have to use puncturing, for fitting 639 bits in radio frames of 600 bits.

\section{The Rate Matching Process}

The receiver can take advantage of the repeated bits in order to improve the error rate. Denoting $P_{S}(\epsilon)$ and $P_{D}(\epsilon)$ respectively the bit error probability without and with bit repetition, then for the situation of $N$ coded bits entering the rate matching unit and considering the addition of $\Delta N$ repeated bits forming a sequence of $N+\Delta N$ bits, the overall bit error probability is given by:

$$
P(\epsilon)=\left(\frac{\Delta N}{N}\right) \cdot P_{D}(\epsilon)+\left(1-\frac{\Delta N}{N}\right) \cdot P_{S}(\epsilon)
$$

As an example, Figure 4 shows the error probability versus $E_{b} / N_{0}$ for different relations of $\Delta N / N$ in a typical BPSK channel strictly subjected to additive Gaussian white noise. There we can clearly see the improvement achieved in $P(\epsilon)$ with the mechanism of bits repetition.

Suppose we have a multimedia transmission consisting of $n$ channels to be multiplexed, one for each media, with the

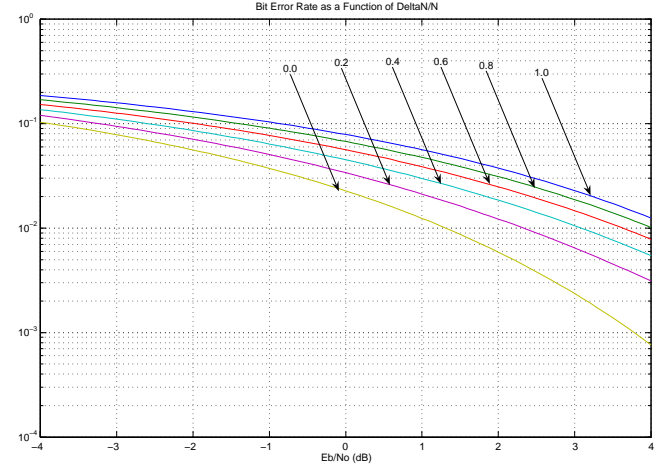

Fig. 4. BER Curves for BPSK Signal Corrupted by Additive Noise

respective QoS objectives, expressed as a certain required value of BER. To cope with these BER values, each channel has to be transmitted with a proper $E_{b} / N_{0}$ ratio. If the physical channel can accommodate more bits than the total of the $n$ channels, it would be possible to distribute these extra bits (spaces) to the channels, duplicating one or more bits in each channel. Therefore it is possible to assign the number of repeated bits in each channel, as a way to manipulate the required $E_{b} / N_{0}$. This is the Rate Matching objective. On the other hand, when the total number of bits surpasses the total bits for the $n$ channels, bits must be removed (punctured) instead. The number of bits to be repeated (or punctured) is given by the upper levels of the protocol.

Unfortunately when a convolutional or turbo encoder is included, there is no closed form for BER calculation, being necessary to resort to computer simulation.

We emphasize that although the examples here presented involve coding and additive white gaussian channel, this approach is by no means limited to this case. On the contrary, any channel model (pedestrian, vehicular, indoor, etc..) that can be simulated or field measured, can provide the BER data that are needed.

Throughout this text we assume that the BER curves are functions of the original number of bits $N$, the number of repeated/punctured bits $\Delta N$ and the value of $E_{b} / N_{0}$. These functions are assumed to be numerically available for all kind of encoders discussed. Appendix I presents one technique capable of generating BER curves from collected BER data.

Having these considerations in mind, a question may be posed: how should we distribute the repetition/puncturing bits among the transport channels in the best possible way. Certainly channels with stringent QoS levels deserve a special attention. On the other way, a precise definition of "best" solution that meet practical requirements should be given.

Next section is devoted to develop a formal solution of this problem. We present an alternative technique to solve it that is fairly simple from the computational viewpoint.

\section{The Optimization Procedure}

Consider the situation where we have $\mathrm{M}$ transport channels. We wish to transmit $N_{i}$ bits per radio frame on the ith-channel 
where the sum $N_{T}=\sum N_{i}$ is different than $N$, the total number of bits that are capable to be transmitted. Hence $\Delta N=$ $N-N_{T}$ bits can be selected for repetition/puncturing, thus reducing/increasing the power requirements without sacrificing QoS. We define as $\Delta N_{i}$ the number of repeated/punctured bits in the ith-channel. Each channel is also characterized by its bit error probability $P_{i}$, a function of $N_{i}, \Delta N_{i}$ and $\left(E_{b} / N_{0}\right)_{i}$. QoS demands require that $P_{i} \leq \alpha_{i}$ where $\alpha_{i}$ is a prefixed value. Since the $N_{i}$ values are known, $P_{i}$ depends only on the two unknowns $\Delta N_{i}$ and $\left(E_{b} / N_{0}\right)_{i}$.

Throughout this work, we will assume that $P_{i}$ is a monotonic decreasing function on both arguments which is a fairly reasonable assumption. For the sake of notation's simplicity, let us define $x_{i}=\Delta N_{i}$ and $y_{i}=\left(E_{b} / N_{0}\right)_{i}$.

These quantities should obey the following restrictions:

1) $x_{i}+N_{i} \geq 0$ and $y_{i} \geq 0$ for all $i \in \overline{1, M}$

2) $x_{1}+x_{2}+\ldots+x_{M}=\Delta N$

3) $P_{i}\left(x_{i}, y_{i}\right) \leq \alpha_{i}$ for all $i \in \overline{1, M}$

Let us define a set $\Omega$ made of all vectors of the form $(\underline{x}, \underline{y})=\left(x_{1}, x_{2}, \ldots, x_{M}, y_{1}, y_{2}, \ldots y_{M}\right)$ that obey the above restrictions. Since this set is not a singleton, one might argue which element of $\Omega$ is the "best".

Among all possible ways of defining a quality criterion, one that seems more suitable for the problem in hand, is the minimization of the maximum $E_{b} / N_{0}$ value that meets the requirements. Hence we can formulate our problem as one of optimization in which we seek the minimization of an artificial variable $w$ subjected to:

1) $y_{i} \leq w$ for all $i \in \overline{1, M}$

2) $(\underline{x}, \underline{y}) \in \Omega$

In the optimization theory parlance, we are faced with an $(2 . M+1)$ dimensional mixed-integer problem with linear objective function but with linear and nonlinear constraints. Problems of this sort tend to require sophisticated computer programs to be solve and to be time consuming.

We will drop for a moment the integrality condition imposed on the $x$ variables. Later we will discuss how this condition should be reinstated.

The main goal of this paper is to show that this problem can be solved by an alternative algorithm that does not require conventional optimization tools at all. On the contrary, this algorithm requires only simple procedures that can be implemented in any computer language with low programming cost and computational effort.

Let us start rewriting the optimization problem in a more convenient way. By defining a new decision variable as $\underline{x}=$ $\left(x_{1}, x_{2}, \ldots, x_{M}, y_{1}, y_{2}, \ldots y_{M}, w\right)$, the problem can be written as:

$$
\min _{\underline{x}} z=f(\underline{x})=w
$$

subjected to:

$$
h(\underline{x})=\sum_{i=1}^{M} x_{i}-\Delta N=0
$$

and for $1<k<M$ :

$$
\begin{aligned}
g_{k}(\underline{x}) & =x_{k}+N_{k} \geq 0 \\
g_{k+M}(\underline{x}) & =w-y_{k} \geq 0 \\
g_{k+2 . M}(\underline{x}) & =\alpha_{k}-P_{k}\left(x_{k}, y_{k}\right) \geq 0
\end{aligned}
$$

Let $\underline{x}^{*}$ a regular point of $\Omega$. The well known theorem of Karush-Kuhn-Tucker [10] states that if $\underline{x}^{*}$ is a local minimum then there exists an scalar $\nu \in \mathbb{R}$ and non-negative vectors $\underline{\mu}, \underline{\lambda}, \underline{\eta} \in \mathbb{R}^{M}$ such that:

$$
\left\{\begin{array}{l}
\nabla f\left(\underline{x}^{*}\right)-\nu \cdot \nabla h\left(\underline{x}^{*}\right)-\underline{\sigma}^{T} \cdot \nabla \underline{g}\left(\underline{x}^{*}\right)=0 \\
\nu \cdot h\left(\underline{x}^{*}\right)=0 \\
\underline{\sigma}^{T} \cdot \underline{g}\left(\underline{x}^{*}\right)=0
\end{array}\right.
$$

where $\underline{\sigma}^{T}=\left[\underline{\mu}^{T}, \underline{\lambda}^{T}, \underline{\eta}^{T}\right]$.

This theorem when applied to the current optimization problem reveals that, besides the fact that $\sum_{i=1}^{M} \lambda_{i}=1$, the following relations must hold for each $k$ in the range $1,2, . ., M$ :

$$
\left\{\begin{array}{l}
-\mu_{k}+\eta_{k} \cdot P_{k x}\left(x_{k}^{*}, y_{k}^{*}\right)-\nu=0 \\
\lambda_{k}+\eta_{k} \cdot P_{k y}\left(x_{k}^{*}, y_{k}^{*}\right)=0 \\
\mu_{k} \cdot\left(x_{k}^{*}+N_{k}\right)=0 \\
\lambda_{k} \cdot\left(w^{*}-y_{k}^{*}\right)=0 \\
\eta_{k} \cdot\left(\alpha_{k}-P\left(x_{k}^{*}, y_{k}^{*}\right)\right)=0 \\
\lambda_{k}, \eta_{k} \geq 0
\end{array}\right.
$$

where $P_{k x}$ and $P_{k y}$ represent the derivatives of function $P_{k}$ respectively in relation to $x$ and $y$.

The important conclusion from the findings shown in Appendix II, is that if $x_{j}^{*}+N_{j}>0$ then $P_{j}\left(x_{j}^{*}, y_{j}^{*}\right)=\alpha_{j}$ and $y_{j}^{*}=w^{*}$. Obviously if $x_{j}^{*}+N_{j}=0$ then $P_{j}\left(x_{j}^{*}, y_{j}^{*}\right) \leq \alpha_{j}$ and $y_{j}^{*} \leq w^{*}$.

From a practical point of view, this optimization problem can be reduced to a one-dimensional search. If we sweep the value of $w^{*}$ on a certain range and if we have means of finding $x_{j}^{*}$ by solving $P_{j}\left(x_{j}^{*}, w^{*}\right)=\alpha_{j}$, then we only need to verify if the condition $\sum_{j=1}^{M} x_{j}^{*}=\Delta N$ holds. The wellknown bisection method is an effective way of implementing this search.

The following procedure was conceived to find the optimal bit allocation:

1) Define $\left[w_{M I N}, w_{M A X}\right]$ as the search region for $w$.

2) Let $w=\left[w_{M I N}+w_{M A X}\right] / 2$

3) For all $j \in \overline{1, M}$ find $x_{j}^{*}$ satisfying $P_{j}\left(x_{j}^{*}, w\right)=\alpha_{j}$

4) Let $S=\sum_{i=1}^{M} x_{i}^{*}$

5) If $S<\Delta N$ then do $w_{M A X}=w$.

6) If $S>\Delta N$ then do $w_{M I N}=w$.

7) If $w_{M A X}-w_{M I N}>$ tolerance then go to 2 .

8) Optimal solution $w^{*}=w$ is found.

At this point it seems appropriate to discuss some facts concerning the feasible region $\Omega$ and the optimal solution. Figure 5 illustrates an example involving 4 transport channels. 
There we can see the xy-plane and all the isocurves associated with the equation $P_{k}(x, y)=\alpha_{k}$. Notice that these curves are compatible with the fact that function $P(x, y)$ is monotonic decreasing in both arguments.

One can conclude from the results obtained that each optimal solution $\left(x_{k}^{*}, y_{k}^{*}\right)$ must always be in the corresponding isocurve. Those solutions in which $x_{k}^{*}+N_{k}>0$ must have $y_{k}^{*}=w^{*}$ but those in which $x_{k}^{*}+N_{k}=0$, the variable $y_{k}$ can have values to the right of the isocurve and to the left of $w^{*}$. The value on the isocurve corresponds to the minimum energy case and therefore should be the one chosen.

In this illustration, the optimal value $w^{*}$ indicates nonzero $\Delta N$ values for transport channels 2 to 4 . Channel 1 does not require extra bits because its isocurve does not intercept the vertical line indicated by $w^{*}$.

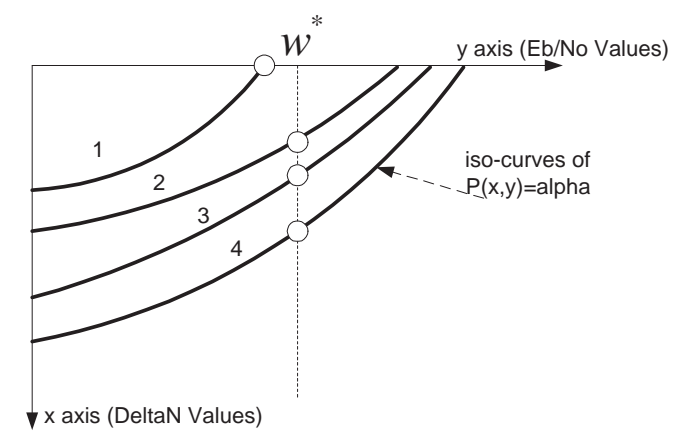

Fig. 5. Feasible region and optimal solution

It is now opportune to discuss how the integrality condition can be reinstated. We are well aware that in mixed-integer programming the optimal solution very often is not the truncated or rounded version of the optimal real solution. Nevertheless, due to the smoothness of function $P(x, y)$, all simulation results have revealed that the variation of this function between two consecutive integers were not superior to $2 \%$. Although we cannot strictly speak of optimality if truncations is the process to produce integer solutions, we are fairly confident that results produced this way will not deviate more than a few per cent of the optimal solution. Therefore, from a practical point of view, this type of solution seems to attend our purposes.

\section{EXAMPLES}

In order to illustrate the proposed methodology, two examples are presented. In the first one, we have four transport channels that share a frame 600 bits long with characteristics described in Table I.

This is the real case already considered at the beginning of Section III, where we have voice transmission at $12.2 \mathrm{kbit} / \mathrm{s}$ by an AMR codec. There are three transport channels (1,2 and 3 ) carrying bit sequences with different QoS requirements and an associated signaling channel (4). A spreading factor of 64 is used, thus resulting in a physical channel of $600 \mathrm{kbit} / \mathrm{s}$. The BER values are those stated in [9].

The function $P(x, y)$ is obtained by means of the methodology described in Appendix I applied on data generated by computer simulation.
If no extra bit is given to any of these transport channels, the minimum $E_{b} / N_{0}$ values that meets the desired BER are respectively, $2.147 \mathrm{~dB}, 1.983 \mathrm{~dB}, 1.733 \mathrm{~dB}$ and $2.612 \mathrm{~dB}$, this last value corresponding to the most severe case. Note that 84 extra bits can be distributed over these channels.

A MATLAB script that implements the allocation bit procedure here discussed was written. When this algorithm is run, the result tell us that 13 extra bits should be given to channel 1 and 74 extra bits should be given to channel 4, thus reducing the maximum $E_{b} / N_{0}$ to the value of $1.982 \mathrm{~dB}$. Consequently, the energy saving for the worst channel is $0.630 \mathrm{~dB}$ but the global savings (sum of savings for all channels) is $0.795 \mathrm{~dB}$. The processing time for this example in a Pentium IV-2.6MHz computer was less than a second.

TABLE I

PARAMETERS OF THE TRANSPORT CHANNELS IN CS MODE

\begin{tabular}{|c|c|c|c|c|c|c|}
\hline Chnl & Coder & Bits & BER & $E_{b} / N_{0}$ & R/P & $E_{b} / N_{0}$ \\
\hline \hline 1 & CC3 & 152 & $5.10^{-4}$ & 2.147 & +13 & 1.982 \\
\hline 2 & CC3 & 167 & $10^{-3}$ & 1.983 & 0 & 1.982 \\
\hline 3 & CC2 & 69 & $5.10^{-3}$ & 1.733 & 0 & 1.982 \\
\hline 4 & CC3 & 129 & $10^{-4}$ & 2.612 & +74 & 1.982 \\
\hline Total & & 516 & & & +84 & \\
\hline
\end{tabular}

In the previous example, only repetition bits are needed to optimize the Rate Matching operation. This second example shows the more general case of using repetition as well as puncturing. For this purpose, refer to the PS mode case also presented in Section III. Considering the second option, where we have 639 bits to be fitted in a frame of 600 bits, Table II shows the results of the algorithm application. It is a rather interesting situation, because, in the contrary of the natural expectation of suppressing (puncture) 39 bits, the algorithm shows the need of simultaneous puncturing and repetition of bits in the two channels.

Note that the value of $E_{b} / N_{0}$ after repetition/puncturing is $8.23 \%$ smaller than the case where the original number of bits is preserved.

TABLE II

PARAMETERS OF THE TRANSPORT CHANNELS IN PS MODE

\begin{tabular}{|c|c|c|c|c|c|c|}
\hline Chnl & Coder & Bits & BER & $E_{b} / N_{0}$ & R/P & $E_{b} / N_{0}$ \\
\hline \hline A & TC & 510 & $5.10^{-4}$ & 1.742 & -45 & 2.529 \\
\hline B & CC3 & 129 & $10^{-4}$ & 2.756 & +6 & 2.529 \\
\hline Total & & 639 & & & -39 & \\
\hline
\end{tabular}

\section{CONCLUSION}

This paper discussed the performance of voice calls in UMTS networks considering some important aspects of the transmission system in CS and PS mode. The structure of the transport channel have been described for both modes and a performance analysis focusing on the rate matching operation have been carried out. In addition, a method has been proposed for allocating/puncturing bits in transport channels of an UMTS communication system in order to meet QoS levels and minimize the maximum $E_{b} / N_{0}$ required in each one of 
these channels. A mathematical analysis of this problem has revealed that this minimization can be produced by a simple algorithm that is easily programmable and computationally cheap. To the best of the author's knowledge, the approach here investigated to optimally allocate repetition bits has not yet been addressed in the technical literature.

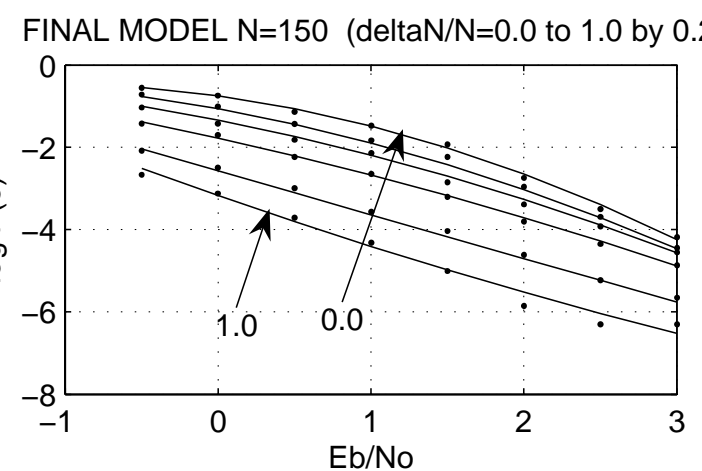

FINAL MODEL N=300 (deltaN/N=0.0 to 1.0 by 0.2 )

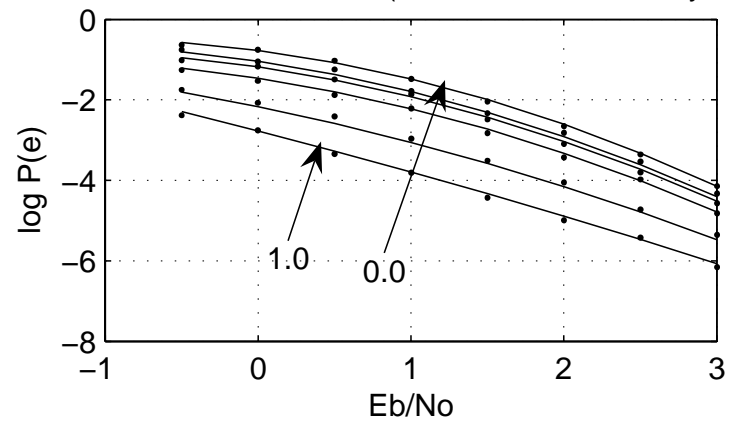

FINAL MODEL N=600 (deltaN/N=0.0 to 1.0 by 0.2 )

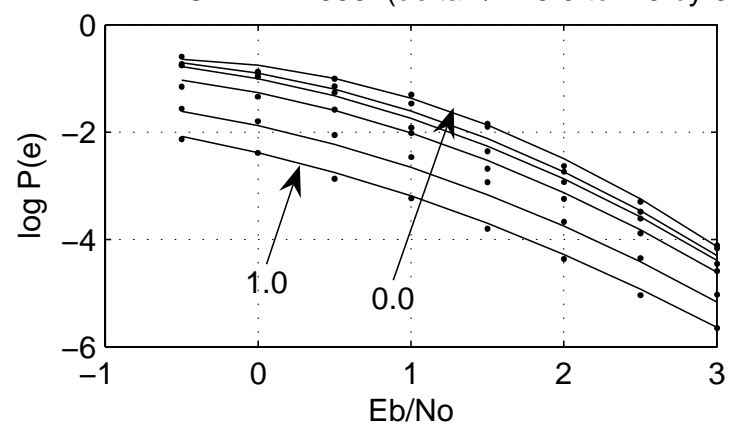

Fig. 6. 1/2 Convolutional Coding Adjusting Model

\section{APPENDIX I}

\section{A Model for the Function $P\left(N, \Delta N, E_{b} / N_{0}\right)$}

Due to the previously discussed decreasing nature of this function, one among many possible models for a suitable approximation function is:

$$
P\left(N, \Delta N, E_{b} / N_{0}\right)=10^{\sum_{i=0}^{N_{1}} a_{i}(N, \Delta N) \cdot\left(E_{b} / N_{0}\right)^{i}}
$$

where $E_{b} / N_{0}$ is expressed in $\mathrm{dB}$. Each one of the $a^{\prime} s$ coefficients can be estimated from data in the LS sense and they can be further modelled by an expression of the form:

$$
a_{i}(N, \Delta N)=\sum_{j=0}^{N_{2}} b_{i j}(N) \cdot(\Delta N)^{j}
$$

Again each one of the coefficients $b^{\prime} s$ was estimated from data in the LS sense and they were further modelled by an expression of the form:

$$
b_{i j}(N)=\sum_{k=0}^{N_{3}} c_{i j k} \cdot N^{k}
$$

Therefore the probability in questions was modelled by:

$$
P\left(N, \Delta N, E_{b} / N_{0}\right)=10^{f\left[N, \Delta N \cdot E_{b} / N_{0}\right]}
$$

where:

$$
f\left[N, \Delta N \cdot E_{b} / N_{0}\right]=\sum_{i=0}^{N_{1}} \sum_{j=0}^{N_{2}} \sum_{k=0}^{N_{3}} c_{i j k} \cdot\left(E_{b} / N_{0}\right)^{i} \cdot(\Delta N)^{j} \cdot N^{k}
$$

Figure 6 shows the results of this model when applied to the rate $1 / 2$ convolutional coding used in the UMTS system. Data were obtained by computer's simulation. The approximation has shown to be fairly acceptable for the following parameter values: $N_{1}=2, N_{2}=3$ and $N_{3}=3$. Although not shown here, similar good results were also obtained for the $1 / 3$ convolutional coding and $1 / 3$ turbo coding, thus indicating that this fitting function seems to be a nice model for fitting data.

\section{AppendiX II}

\section{Proof of the Equivalent Optimization Condition}

Since physical evidence allows us to believe that function $P$ is monotonic in both arguments, the derivatives mentioned above are strictly negative in the region of interest. Hence $\lambda_{k}>0 \Longrightarrow \eta_{k}>0$.

Let $\Omega_{\lambda}$ be the subset of $\{1,2, . ., M\}$ in which $i \in \Omega_{\lambda} \Longrightarrow$ $\lambda_{i}>0$ (and consequently $\eta_{i}>0$ ). Note that this set is nonempty because the sum of $\lambda^{\prime} s$ is one. Therefore if $j \in \Omega_{\lambda}$ then $y_{j}^{*}=w^{*}$ and $P_{j}\left(x_{j}^{*}, y_{j}^{*}\right)=\alpha_{j}$. Consequently $\nu+\mu_{j}<0$ thus implying that $\nu<0$.

Now let us analyze those $j^{\prime} s$ that do not belong to $\Omega_{\lambda}$. In this case, $\eta_{j}=0$, implying that $\nu+\mu_{j}=0$. Since $\nu<0$, we conclude that $\mu_{j}>0$, which leads to $x_{j}^{*}+N_{j}=0$.

\section{REFERENCES}

[1] Holma H., Toskala A., "WCDMA for UMTS," John Wiley \& Sons,Ltd., England, 3rd. Edition, 2004.

[2] Ojanperã T., Prasad R., "Towards IP Mobility and Mobile Internet," Artech House Universal Personal Communications Series, London, 2001.

[3] Plasse D., "Call Control Scenarios in the "all-IP" UMTS Core Network," 11th International Symposium on Personal Indoor and Mobile Radio Communications, PIRMC2000, Vol. 1, pp. 322-326, Sept. 2000. 
[4] Daniel W., Vijay K.V., "Supporting Real-Time IP Multimedia Services in UMTS," IEEE Communications Magazine, Vol. 41, No. 11, pp. 148155, Nov. 2003.

[5] Yi-Bing L., Ai-Chun P., Yieh-Ran H., "An All-IP Approach for UMTS Third-Generation Mobile Networks," IEEE Network, Vol. 16, No. 5, pp. 8-19, Sept/Oct. 2002.

[6] Baey S., Dumas M., Dumas M.C., "QoS Tuning and Resource Sharing for UMTS WCDMA Multiservice Mobile," IEEE Transactions on Mobile Computing, vol. 1, no. 3, pp. 221-235, July-Sept 2002.

[7] Aftelak S, Bhatoolaul X., "Rate Matching Attribute Settings and Error Rate Performance Sensitivity for Selected UMTS FDD Services," IEEE Semiannual Vehicular Technoogy Conference, October 6-9 2003.

[8] 3GPP Documentation, "Multiplexing and Channel Coding," TS 25.212 v6.2.0, June 2004.

[9] 3GPP Documentation, "Common Test Environment for User Equipment (UE), Conformance Testing," TS 34.108 v5.1.0, June 2004.

[10] Bertsekas D.P., "Nonlinear Programming," Athenas Scientific, 1995.

[11] Sohn I., Bang S.C., "Performance Studies of Rate Matching for WCDMA Mobile Receiver," VCT 2000 Conference, pp. 2661-2665, 2000.

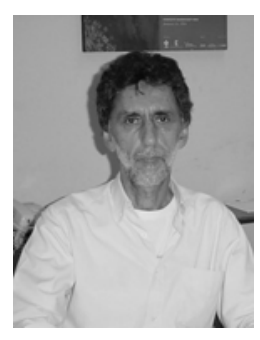

João Célio Brandão received an MSc. degree in Electrical Engineering from the Pontifical Catholic University of Rio de Janeiro (PUC-Rio), Brazil, in 1973. Since then he has worked at the Center for Studies in Telecommunications of PUC-Rio (CETUC) where he is presently Professor and ViceDirector. At CETUC, he has been involved with teaching and research in the area of digital transmission and network performance with particular application to satellite and cellular systems.

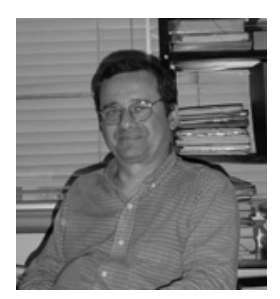

Marco Antonio Grivet Mattoso Maia was born in Rio de Janeiro, Brazil, in 1951. He received the B.S degree in 1974 and the M.Sc. degree in 1977 from the Pontifical Catholic University of Rio de Janeiro (PUC-RIO), Brazil, both in telecommunications engineering. He received the Ph.D. degree in 1983 from the University of Kent, Canterbury, England. He was associate lecturer at the Military Institute of Engineering from 1983 to 1987 and presently is at the Center for Studies in Telecommunications of PUC-RIO. His current research interests include mobile communication, location-based systems, image recovery and visualization and digital image processing.

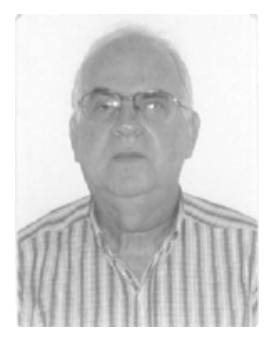

Eduardo Rodrigues Vale was born in Rio de Janeiro, Brazil, in 1949. He received the B.S. degree in 1972, the M.Sc. degree in 1975 and the Ph.D. degree in 2006, all of them from the Pontifical Catholic University of Rio de Janeiro (PUC-RIO), Brazil, in Telecommunications Engineering. Presently he is a Professor in the Telecommunications Engineering Department of Universidade Federal Fluminense (UFF). His current research interests include the area of mobile communications, particularly the Third Generation UMTS systems. 